\title{
Autoionization and ultrafast relaxation dynamics of highly excited states in $\mathrm{N}_{2}$
}

\author{
M. Lucchini, ${ }^{1}$ K. Kim, ${ }^{2}$ F. Calegari, ${ }^{1}$ F. Kelkensberg, ${ }^{3}$ W. Siu, ${ }^{3}$ G. Sansone, ${ }^{1}$ M. J. J. Vrakking, ${ }^{4}$ M. Hochlaf, ${ }^{5}$ and M. Nisoli ${ }^{1}$ \\ ${ }^{1}$ Department of Physics, Politecnico di Milano, National Research Council of Italy, Institute of Photonics and Nanotechnologies (CNR-IFN), \\ Piazza L. da Vinci 32, 20133 Milano, Italy \\ ${ }^{2}$ Department of Physics and Coherent X-ray Research Center (CXRC),Korea Advanced Institute of Science and Technology (KAIST), \\ Daejeon 305-701, Korea \\ ${ }^{3}$ FOM Institute AMOLF, Science Park 104, NL-1098 XG Amsterdam, The Netherlands \\ ${ }^{4}$ Max-Born-Institut, Max Born Strasse 2A, D-12489 Berlin, Germany \\ ${ }^{5}$ Université Paris-Est, Laboratoire Modélisation et Simulation Multi-Echelle, MSME UMR 8208 CNRS, 5 bd Descartes, 77454 \\ Marne-la-Vallée, France
}

(Received 23 May 2012; published 2 October 2012)

\begin{abstract}
We have used the velocity-map imaging (VMI) technique to measure autoionization dynamics in molecular nitrogen initiated by a train of attosecond pulses. The pump-probe measurements show clear evidence of a crossing between potential energy curves of the highly excited $\mathrm{N}_{2}{ }^{+}$ion and of the $\mathrm{N}_{2}{ }^{2+}$ ion. It is found that the autoionization becomes energetically allowed when the two nuclei are still very close $(\sim 3 \AA)$, in contrast with observations in other diatomic molecules, and that it can be coherently manipulated by a strong femtosecond infrared pulse.
\end{abstract}

DOI: 10.1103/PhysRevA.86.043404

PACS number(s): $32.80 . \mathrm{Rm}, 42.65 . \mathrm{Ky}, 42.65 . \mathrm{Re}$

\section{INTRODUCTION}

Ultrafast extreme ultraviolet (XUV) spectroscopy, with temporal resolution in the femtosecond and attosecond ranges, has proven to be an important tool for the investigation of correlated electronic dynamics in atoms and molecules [1-3]. In this context, understanding the coupling of electronic and nuclear degrees of freedom as well as the electron correlations immediately following, or accompanying, optical excitation is particularly important and requires the use of ultrafast methods. The sudden removal of an electron from an inner-valence shell of an atom or molecule by the absorption of high-energy photons can give rise to ultrafast electronic relaxation processes [4], including intra-atomic or intramolecular autoionization, interatomic or intermolecular Coulombic decay (ICD) [5], and additional relaxation processes, which involve nuclear dynamics, generally on a longer temporal scale. Autoionization can be a very efficient electronic decay process, evolving on a femtosecond temporal scale, where electron correlation effects play a crucial role [6]. The investigation of autoionization in molecules gives access not only to the time evolution of correlated electronic motion but also to the possibility to influence the nuclear motion by acting on the electron interaction, thus offering new insight into non-Born-Oppenheimer dynamics.

Because of the wide interest in molecular dynamics initiated by XUV photoionization, many experimental techniques have been implemented. Since the relaxation dynamics of highly excited states in molecules can be very fast, the use of femtosecond or attosecond techniques is required if one wishes to time-resolve the process. Isolated attosecond pulses and trains of attosecond pulses have recently been used for the investigation of electron dynamics in molecules. Subfemtosecond electron localization after attosecond excitation was observed in $\mathrm{H}_{2}$ and $\mathrm{D}_{2}$ molecules [7], and control of photoionization of $\mathrm{D}_{2}$ and $\mathrm{O}_{2}$ molecules was achieved by using attosecond pulse trains (APT's) [8,9]. Attosecond pulse trains have also been applied to study the influence of the excitation of vibrational levels of autoionizing states close to the first ionization threshold of $\mathrm{N}_{2}[10,11]$. Femtosecond XUV pulses generated by high harmonic generation (HHG) have been implemented to measure fast dynamics in $\mathrm{N}_{2}$ molecules after the creation of highly excited states [3] as well as to measure autoionization processes in $\mathrm{O}_{2}$ molecules [12].

In this work $\mathrm{N}_{2}$ molecules were photoionized with APT's and the subsequent ultrafast relaxation dynamics was probed using strong infrared (IR) pulses. By using the velocity-map imaging (VMI) technique the onset of autoionization of the molecular ion after $120 \mathrm{fs}$ and at an internuclear distance of only $\sim 3 \AA$ was observed, thus demonstrating extreme temporal and spatial resolution, and showing that in dissociating $\mathrm{N}_{2}{ }^{+}$ molecules, autoionization become important at internuclear distances that are an order of magnitude shorter than was encountered, with different techniques, in $\mathrm{O}_{2}$ [12] and $\mathrm{CO}$ [13].

\section{RESULTS AND DISCUSSION}

In the experiment, infrared pulses with 15 -fs time duration and 800-nm central wavelength were used to generate an APT by HHG in a 2.5-mm-thick cell filled with krypton or xenon gas at a static pressure ( $~ 5$ Torr). The XUV pulses were focused onto a target consisting of $\mathrm{N}_{2}$ molecules. The bandwidth of the XUV radiation extends far beyond the ionization threshold of $\mathrm{N}_{2}$ at $15.58 \mathrm{eV}$ [14], for both generating media. Figure 1 shows the potential energy curves of the relevant electronic states of $\mathrm{N}_{2}{ }^{+}$[15] and $\mathrm{N}_{2}{ }^{2+}$ [16]. In the case of APT's generated in $\mathrm{Kr}$, the harmonic spectrum extends up to $\sim 55 \mathrm{eV}$. In this case the XUV photons have enough energy to leave the molecule in a highly excited electronic state of $\mathrm{N}_{2}{ }^{+}$or in a low-excited state of $\mathrm{N}_{2}{ }^{2+}$ (double ionization threshold: $42.88 \mathrm{eV}$ [17]). By contrast, the maximum photon energy of the APT generated in $\mathrm{Xe}$ is below the double ionization threshold, so that in this case the molecules are excited to a manifold of excited states of $\mathrm{N}_{2}{ }^{+}$. By using a velocity-map imaging spectrometer $[18,19]$ we measured the kinetic energy and the angular distribution of 
(a)

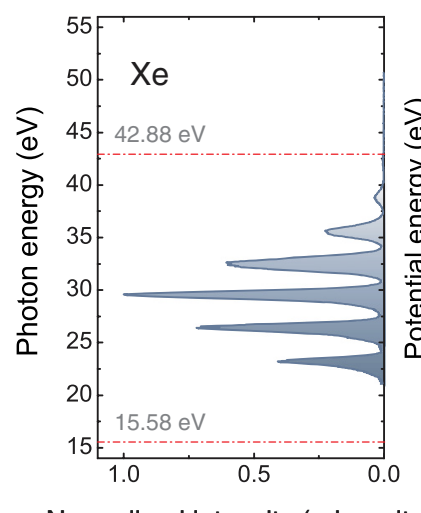

(b)

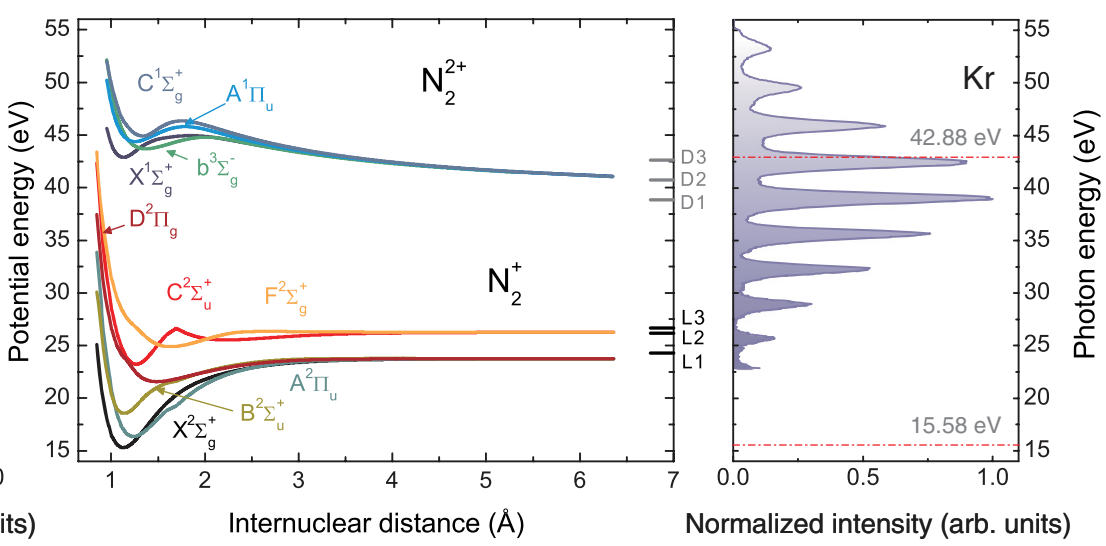

(c)

FIG. 1. (Color online) Spectra of the XUV pulses produced by HHG in (a) xenon and (c) krypton. (b) Potential energy curves of the relevant electronic states of $\mathrm{N}_{2}^{+}[15]$ and $\mathrm{N}_{2}^{2+}[16]$ measured starting from the $\mathrm{N}_{2}\left(\mathrm{X}^{1} \Sigma_{g}^{+}, v=0\right)$ state.

the $\mathrm{N}^{+}$ions resulting from the dissociative ionization and/or Coulomb explosion induced by the XUV pulses. The total $\mathrm{N}^{+}$yield as a function of the kinetic energy release (KER), defined as the energy of one single $\mathrm{N}^{+}$fragment, is obtained by angular integration of the retrieved momentum distribution and it is shown in Figs. 2(a) and 2(b).

In the case of excitation by APT's generated in $\mathrm{Kr}$ [solid curve in Fig. 2(a)], three main spectral features are present: (i) a sequence of closely spaced peaks at a KER below $1 \mathrm{eV}$, (ii) a band peaked at $\sim 0.9 \mathrm{eV}$, and (iii) a broad band with a peak at $\sim 2.3 \mathrm{eV}$. In the case of excitation by APT's generated in Xe [solid curve in Fig. 2(b)], the peak sequence below $1 \mathrm{eV}$ is similar to the one measured when using $\mathrm{Kr}$, but the band at $\sim 0.9 \mathrm{eV}$ is much weaker and the high-energy band is replaced by a small tail extending up to $\sim 4 \mathrm{eV}$. The closely spaced peaks below $1 \mathrm{eV}$ can be assigned to predissociation of vibrational levels $\left(v^{+} \geqslant 3\right)$ of the $\mathrm{N}_{2}{ }^{+}\left(C^{2} \Sigma_{u}^{+}\right)$electronic state [14,20-22]. The band peaked at $\sim 0.9 \mathrm{eV}$ originates from direct dissociation of the $\mathrm{N}_{2}{ }^{+}\left(F^{2} \Sigma_{g}^{+}\right)$state to the third dissociation limit L3 [15]. The increased relative intensity of the $0.9-\mathrm{eV}$ peak in the case of APT's generated in $\mathrm{Kr}$ is due to the fact that the $\mathrm{N}_{2}{ }^{+}\left(F^{2} \Sigma_{g}^{+}\right)$state is relatively more strongly populated in this case, since a larger fraction of the harmonic photons has sufficient energy to populate this state. The signal at kinetic energies $>2 \mathrm{eV}$ originates from some of the many highly excited states of $\mathrm{N}_{2}{ }^{+}$that exist at high energy or from low excited states of $\mathrm{N}_{2}{ }^{2+}$. Since this signal is not present when $\mathrm{N}_{2}$ is excited by APT's generated in Xe, these electronic states have energies close to or even higher than the second ionization threshold. The $\mathrm{N}_{2}{ }^{2+}$ ground state is a long-living metastable state that does not dissociate in a time window $0-15 \mu \mathrm{s}$ [23], so that it is not expected to produce significant $\mathrm{N}^{+}$fragments in the 200-ns time gate applied to the detector. On the other hand, the $\mathrm{N}_{2}{ }^{2+}\left(A^{1} \Pi_{u}\right)$ and the $\mathrm{N}_{2}{ }^{2+}$ $\left(C^{1} \Sigma_{g}^{+}\right)$states (following the notation presented in Ref. [24]) can produce ions in the kinetic energy region of interest. Since it is known that spin-conserving transitions are in general more intense than spin-nonconserving ones we did not consider $\mathrm{N}_{2}{ }^{2+}$ triplet states, such as the $\mathrm{N}_{2}{ }^{2+}\left(b^{3} \Sigma_{g}^{-}\right)$. Finally, as will be discussed below, we show that the $2.3-\mathrm{eV}$ feature in our experiments is primarily due to autoionization of $\mathrm{N}_{2}{ }^{+}$.

\section{A. Pump-probe measurements}

We used a 15 -fs IR pulse, with a peak intensity of $3-4 \times$ $10^{12} \mathrm{~W} / \mathrm{cm}^{2}$, to probe the molecular dissociation dynamics initiated by APT's generated in Kr: As shown in Fig. 3, the

(a)

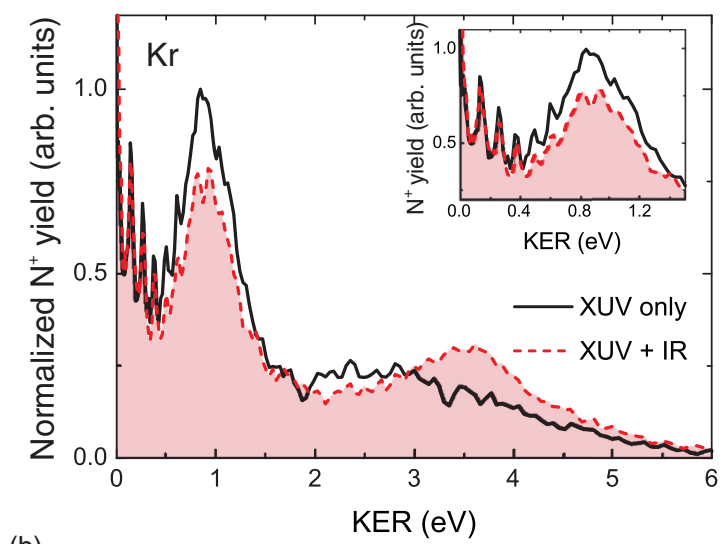

(b)

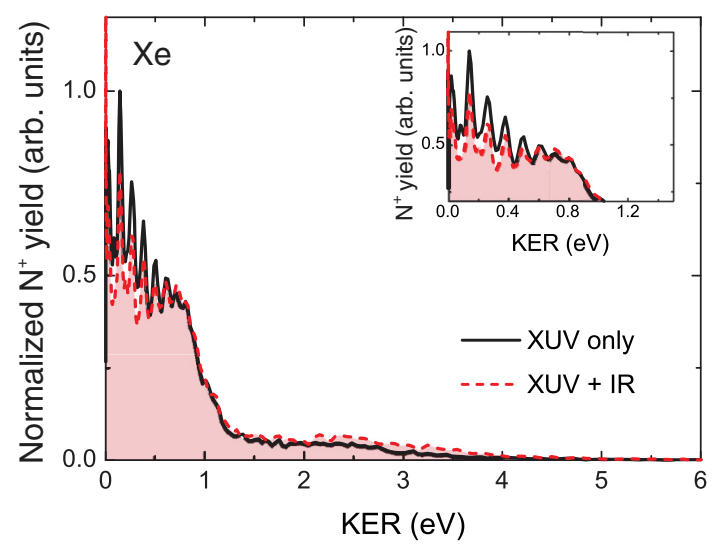

FIG. 2. (Color online) $\mathrm{N}^{+}$kinetic energy distributions with only the XUV pump pulse present (solid lines) and with both pump and IR probe pulses at zero time delay (dashed lines and shaded area) in the case of XUV pulses generated in krypton (a) and in xenon (b). The insets show the low-energy portion of the $\mathrm{N}^{+}$kinetic energy distributions. 


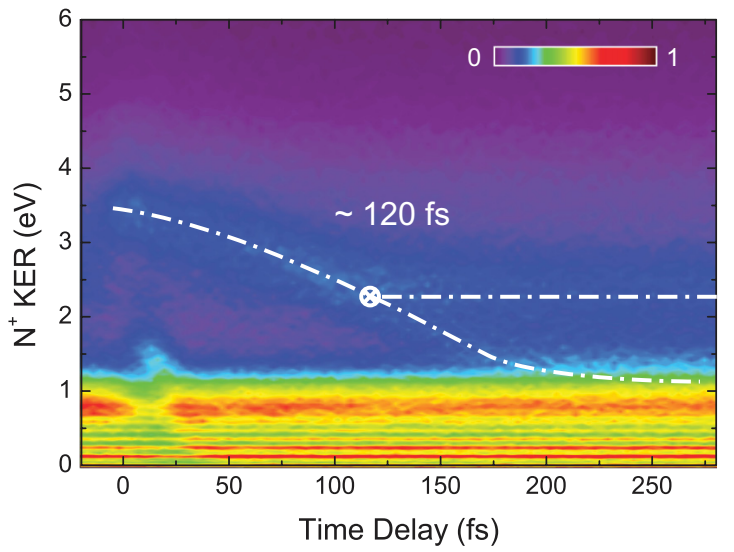

FIG. 3. (Color online) $\mathrm{N}^{+}$kinetic energy distributions as functions of the delay between the APT generated in $\mathrm{Kr}$ and the infrared probe pulse for fragments ejected at $0 \pm 25^{\circ}$. Colour scale shows fragment yield in arbitrary units.

presence of the IR pulse leads to the introduction of a new high-energy feature that extends to about $3.7 \mathrm{eV}$ when the IR and XUV pulses are overlapped in time, and that decreases toward lower energies when the delay between the XUV pulse and the IR pulse is increased. Importantly, for delay times below 120 fs the IR pulse inhibits the observation of the $2.3-\mathrm{eV}$ kinetic energy feature described in the preceding paragraph. This behavior is indicated by the white dash-dotted lines in Fig. 3. These observations are due to an indirect double ionization process, as explained in Fig. 4. The XUV photons populate a superexcited state of the molecular ion, $\left(\mathrm{N}_{2}{ }^{+}\right)^{*}$, which can have an internal energy above the double photoionization threshold $[24,25]$. If there is no IR pulse present in the experiment (or if the delay between the XUV and the IR pulses is long enough), the excited wave packet evolves along the $\left(\mathrm{N}_{2}{ }^{+}\right)^{*}$ potential energy curve until the internuclear distance is sufficient to energetically allow the autoionization

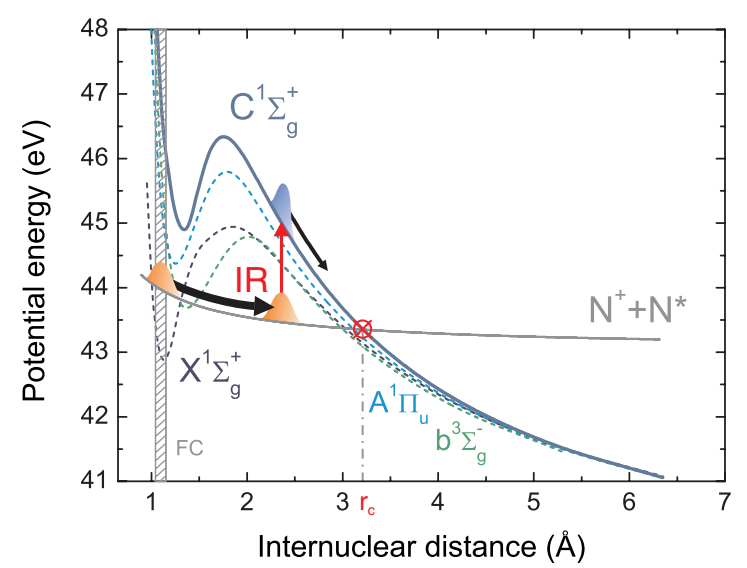

FIG. 4. (Color online) Schematic of the mechanism leading to the population of the $\mathrm{N}_{2}^{2+}\left(C^{1} \Sigma_{g}^{+}\right)$electronic state via the $\left(\mathrm{N}_{2}^{+}\right)^{*}$ autoionizing state involved in the relaxation process described in the text. The potential energy curve of $\left(\mathrm{N}_{2}^{+}\right)^{*}$ (gray solid line) is qualitatively drawn assuming a non-bonding potential and a flat longrange behavior, with an aymptotic energy of $2.3 \mathrm{eV}$ (i.e., $4.6 \mathrm{eV}$ above the first dissociation limit D1 of the dication, located at $38.84 \mathrm{eV}$ above the $\mathrm{N}_{2}$ ground state). process, which gives rise to the band peaked at $2.3 \mathrm{eV}$ measured in the $\mathrm{N}^{+}$kinetic energy spectra in the absence of the IR probe pulse. The autoionization of $\left(\mathrm{N}_{2}{ }^{+}\right)^{*}$ can only occur starting at the internuclear distance where the $\left(\mathrm{N}_{2}{ }^{+}\right)^{*}$ curve lies above the double ionization curve (see Fig. 4). Therefore the autoionization can be inhibited by exposing the molecule to an IR pulse at short time delays. Indeed, Fig. 3 clearly shows that the band peaked at $2.3 \mathrm{eV}$ disappears in the temporal window between $\tau=0$ and $\tau \sim 120$ fs. In this time window the IR pulse can ionize the dissociating $\mathrm{N}_{2}{ }^{+}$ molecular ion, populating $\mathrm{N}_{2}{ }^{2+}$ states that undergo direct dissociation producing two $\mathrm{N}^{+}$fragments. The kinetic energy of these fragments depends on the internuclear distance at which the transition to the dication state takes place, and hence, on the XUV-IR time delay. Indeed, the signal coming from this two-color dissociation relaxes toward lower kinetic energies for longer time delays, following the potential energy curve of the final dication state [26]. Since the maximum energy of these $\mathrm{N}^{+}$fragments, measured at $\tau=0$, is $\sim 3.7 \mathrm{eV}$, the final $\mathrm{N}_{2}{ }^{2+}$ state can be either the $\mathrm{N}_{2}{ }^{2+}\left(C^{1} \Sigma_{g}^{+}\right)$or the $\mathrm{N}_{2}{ }^{2+}\left(A^{1} \Pi_{u}\right)$ states, which directly dissociate to the $\mathrm{D} 1: \mathrm{N}^{+}\left({ }^{3} \mathrm{P}\right)+\mathrm{N}^{+}\left({ }^{3} \mathrm{P}\right)$ limit located at $38.84 \mathrm{eV}$ above the $\mathrm{N}_{2}$ ground state [27]. A $\mathrm{N}_{2}{ }^{2+}\left(A^{1} \Pi_{u}\right)$ contribution can be excluded, however, since it is known to produce two $\mathrm{N}^{+}$fragments with an asymmetry parameter $\beta_{2}<0$ (ion fragments predominantly ejected in the direction perpendicular to the laser polarization) [28], in contrast to the measured value $\beta_{2} \simeq 1$ (ion fragments predominantly ejected in the parallel direction). Indeed, as demonstrated by Jiang et al. using two-photon ionization of $\mathrm{N}_{2}$ with 44-eV photons, the population of the $\mathrm{N}_{2}{ }^{2+}\left(A^{1} \Pi_{u}\right)$ states leads to emission of $\mathrm{N}^{+}$ions in the direction perpendicular to the laser polarization [28]. In our case the fragments are mainly detected along the laser polarization direction, clearly indicating the different symmetry of the final $\mathrm{N}_{2}{ }^{2+}$ state in our measurement. It is noteworthy that the experimental results shown in Fig. 3 reveal a persistence of IR laser-assisted ionization over the entire time delay range. For $\tau<120 \mathrm{fs}$, the role of the IR is obvious, since the molecule lacks the energy to autoionize, and the absorption of one or more IR photons is necessary to reach the dication potential energy curve. For $\tau>120 \mathrm{fs}$, however, when autoionization has become allowed, the two-color ionization process persists, as evidenced by the continued observation of a dependence of the KER on the delay between the XUV and IR pulses. This is a clear indication that, in addition to the autoionization mechanism, which does not involve the IR laser, the interaction of the electric field of the IR pulse with the correlated electron wave function of the highly excited state of the cation leads to an enhancement of the population of the dication state.

\section{B. Time-to-internuclear distance mapping}

As a major result of the imaging technique used in the experiments, the kinetic energy map of Fig. 3 directly shows when the autoionizing state intersects the $\mathrm{N}_{2}{ }^{2+}\left(C^{1} \Sigma_{g}^{+}\right)$state. Indeed, for $\tau>120 \mathrm{fs}$ the band at $2.3 \mathrm{eV}$ and the band associated with the $\mathrm{N}_{2}{ }^{2+}\left(C^{1} \Sigma_{g}^{+}\right)$state are both present. The kinetic energy map can be seen as a precise clock, which measures, with femtosecond temporal resolution, when autoionization starts to be an energetically allowed process. 
The time-to-internuclear distance mapping is then obtained by calculating the potential energy curves of the dication, even without a detailed knowledge of the potential energy curve of the autoionizing state. Indeed, following the calculated $\mathrm{N}_{2}{ }^{2+}$ $\left(C^{1} \Sigma_{g}^{+}\right)$curve, it is possible to obtain an estimate of the internuclear distance $r_{c}$ at which the potential energy curves intersect. The internuclear distance at which a sharing of the Coulomb potential energy between the two $\mathrm{N}^{+}$ions leads to a kinetic energy of $2.3 \mathrm{eV}$ for each fragment can easily be calculated to be $3.2 \AA$. Therefore, the process of autoionization in $\mathrm{N}_{2}$ does not require a large internuclear separation of the fragments, as reported, for example, in $\mathrm{O}_{2}$ [12] and in $\mathrm{CO}$ [13]. On the contrary, it can occur at internuclear distances typical for Interatomic Coulombic Decay (ICD) in van der Waals dimers, where autoionization occurs when the atoms are in close proximity $[5,29]$.

\section{OUTLOOK}

Ultrafast dynamics in molecules initiated by sudden absorption of XUV or soft-X-ray photons, which is at the basis of a number of important phenomena in nature, can reveal a high degree of complexity. Generally many-body effects are triggered, where electron correlations play a crucial role.
The imaging technique applied in this work to $\mathrm{N}_{2}$ molecules, in combination with few-femtosecond or sub-femtosecond XUV pulses, can be extended to the investigation of the autoionization process in complex molecular systems. This is particularly interesting due to the importance of the autoionization mechanisms both from the applied and theoretical points of view. Indeed, autoionization following XUV excitation leads to the generation of electrons and ions, thus constituting an important mechanism for radiation damage of molecules exposed to XUV radiation.

\section{ACKNOWLEDGMENTS}

The research leading to the results presented in this paper has received funding from the European Research Council under the European Community's Seventh Framework Programme (FP7/2007-2013)/ERC grant agreement No. 227355-ELYCHE. We acknowledge financial support from the Italian Ministry of Research (FIRB-IDEAS RBID08CRXK), and support from European Union within Contract No. 228334 JRA-ALADIN (Laserlab Europe II) and from MC-RTN ATTOFEL (FP7-238362). We thank Y. Hikosaka and K. Ito for providing us with the $\mathrm{N}_{2}{ }^{+}$potential energy curves and $\mathrm{M}$. Tommasini for helpful discussions.
[1] A. S. Sandhu and X. M. Tong, IEEE J. Select. Top. Quantum Electr. 18, 351 (2012).

[2] F. Krausz and M. Ivanov, Rev. Mod. Phys. 81, 163 (2009).

[3] E. Gagnon, P. Ranitovic, X. Tong, C. L. Cocke, M. M. Murnane, H. C. Kapteyn, and A. S. Sandhu, Science 317, 1374 (2007).

[4] F. Remacle and R. D. Levine, Proc. Natl. Acad. Sci. 103, 6793 (2006).

[5] L. S. Cederbaum, J. Zobeley, and F. Tarantelli, Phys. Rev. Lett. 79, 4778 (1997).

[6] N. Moiseyev and L. S. Cederbaum, J. Phys. B 32, L279 (1999).

[7] G. Sansone et al., Nature (London) 465, 763 (2010).

[8] F. Kelkensberg et al., Phys. Rev. Lett. 107, 043002 (2011).

[9] W. Siu et al., Phys. Rev. A 84, 063412 (2011).

[10] S. Haessler et al., Phys. Rev. A 80, 011404 (2009).

[11] J. Caillat, A. Maquet, S. Haessler, B. Fabre, T. Ruchon, P. Salières, Y. Mairesse, and R. Taïeb, Phys. Rev. Lett. 106, 093002 (2011).

[12] A. S. Sandhu, E. Gagnon, R. Santra, V. Sharma, W. Li, P. Ho, P. Ranitovic, C. L. Cocke, M. M. Murnane, and H. C. Kapteyn, Science 322, 1081 (2008).

[13] T. Osipov et al., Phys. Rev. A 81, 011402 (2010).

[14] P. Baltzer, M. Larsson, L. Karlsson, B. Wannberg, and M. Carlsson Göthe, Phys. Rev. A 46, 5545 (1992).

[15] T. Aoto, K. Ito, Y. Hikosaka, A. Shibasaki, R. Hirayama, N. Yamamono, and E. Miyoshi, J. Chem. Phys. 124, 234306 (2006).
[16] A. Ben Houria and M. Hochlaf (private communication)

[17] M. Hochlaf, R. I. Hall, F. Penent, H. Kjeldsen, P. Lablanquie, M. Lavollée, and J. H. D. Eland, Chem. Phys. 207, 159 (1996).

[18] A. T. J. B. Eppink and D. H. Parker, Rev. Sci. Instrum. 68, 3477 (1997).

[19] O. Ghafur, W. Siu, P. Johnsson, M. F. Kling, M. Drescher, and M. J. J. Vrakking, Rev. Sci. Instrum. 80, 033110 (2009).

[20] J. H. D. Eland and E. J. Duerr, Chem. Phys. 229, 13 (1998).

[21] C. Nicolas, C. Alcaraz, R. Thissen, M. Vervloet, and O. Dutuit, J. Phys. B 36, 2239 (2003).

[22] M. Hochlaf, G. Chambaud, and P. Rosmus, J. Phys. B 30, 4509 (1997); 31, 4059 (1998) [corrigendum].

[23] M. J. Besnard, L. Hellner, G. Dujardin, and D. Winkoun, J. Chem. Phys. 88, 1732 (1988).

[24] M. Ahmad, P. Lablanquie, F. Penent, J. G. Lambourne, R. I. Hall, and J. H. D. Eland, J. Phys. B 39, 3599 (2006).

[25] A. Ehresmann et al., J. Phys. B 33, 473 (2000).

[26] H. Stapelfeldt, E. Constant, and P. B. Corkum, Phys. Rev. Lett. 74, 3780 (1995).

[27] R. W. Wetmore and R. K. Boyd, J. Phys. Chem. 90, 5540 (1986).

[28] Y. H. Jiang et al., Phys. Rev. Lett. 102, 123002 (2009).

[29] T. Jahnke et al., Phys. Rev. Lett. 93, 163401 (2004). 